\title{
Cx43 reverses the resistance of A549 lung adenocarcinoma cells to cisplatin by inhibiting EMT
}

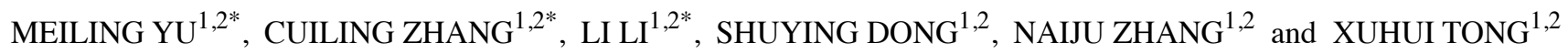 \\ ${ }^{1}$ Department of Pharmacy, The First Affiliated Hospital of Bengbu Medical College, Anhui, Bengbu 233004; \\ ${ }^{2}$ Faculty of Pharmacy, Bengbu Medical College, Anhui, Bengbu 233030, P.R. China
}

Received February 19, 2014; Accepted March 13, 2014

DOI: $10.3892 /$ or.2014.3163

\begin{abstract}
Cisplatin (CDDP) is one of the standard first-line chemotherapeutic agents for advanced non-small cell lung cancer (NSCLC). Unfortunately, prolonged exposure to CDDP results in acquired resistance which prevents the successful treatment of lung cancer patients. Thus, it is necessary to explore the mechanism underlying the resistance of NSCLC to CDDP. In the present study, a CDDP-resistant human lung cancer cell line A549/CDDP was established from the parental cell line A549. The results demonstrated that A549/CDDP cells acquired an epithelial-mesenchymal transition (EMT) phenotype, with morphological changes including acquisition of a spindle-like fibroblastic phenotype, downregulation of E-cadherin, upregulation of mesenchymal markers (vimentin, Snail and Slug), and increased capability of invasion and migration. Compared with A549 cells, the A549/CDDP cells showed decreased connexin43 (Cx43) expression. Overexpression of Cx43 reversed EMT and CDDP resistance in the A549/CDDP cells. Conversely, knockdown of Cx43 expression by siRNACx43 initiated EMT and induced CDDP insensitivity in A549 cells. In summary, Cx43 reverses CDDP resistance in A549 CDDP-resistant cells by preventing EMT, making Cx43 a possible therapeutic target for lung cancer.
\end{abstract}

\section{Introduction}

Lung cancer is the leading cause of death from cancer in China. Non-small cell lung cancer (NSCLC) constitutes $\sim 85 \%$ of all lung cancers (1), with adenocarcinoma comprising the major histological type. Platinum-based chemotherapeutic agents are the standard first-line chemotherapeutic agents for advanced NSCLC (2). Cisplatin (CDDP), for example, is widely used

Correspondence to: Dr Xuhui Tong, Faculty of Pharmacy, Bengbu Medical College, 2600 Donghai Road, Anhui, Bengbu 233004, P.R. China

E-mail: xuhuitong409@sohu.com

*Contributed equally

Key words: cisplatin, A549, Cx43, epithelial-mesenchymal transition, lung cancer for the treatment of NSCLC. However, acquired resistance develops during treatment, leading to tumor recurrence and further progression. Therefore, understand the underlying mechanism of drug resistance to CDDP and increasing the sensitivity to therapeutic drugs are key steps towards the improved treatment of lung cancer patients.

Studies suggest a direct molecular and phenotypic association between resistance to chemotherapy and acquisition of epithelial-mesenchymal transition (EMT) characteristics in cancer cells (3-6). EMT is a cellular process during which epithelial polarized cells become motile mesenchymalappearing cells (7-9) and gain increased cell motility and invasiveness $(10,11)$. EMT involves loss of epithelial cellcell junctions and expression of epithelial markers such as E-cadherin, and gain in the expression of mesenchymal markers such as vimentin, Snail and Slug.

Connexins are a group of homologous proteins that form the inter-membrane channels of gap junctions (12). An abnormal expression level and distribution of connexins are closely related to tumor formation $(13,14)$. Studies indicate that Cx genes, including $\mathrm{Cx} 43$, are tumor-suppressor genes $(15,16)$. $\mathrm{Cx} 43$ is one of the most common of the connexins and the major Cx homolog expressed in lung tissue (17-19). Studies have shown that $\mathrm{Cx} 43$ plays important roles in cancer development, cell proliferation, apoptosis, invasion and metastasis in lung cancer (20-26). Most importantly, Cx43 is able to sensitize NSCLC cells to CDDP and ionizing radiation $(27,28)$.

In the present study, a CDDP-resistant human lung adenocarcinoma A549 cell line (A549/CDDP) was established. We found that $\mathrm{Cx} 43$ is involved in the acquisition of EMT in A549/ CDDP cells. By overexpression of Cx43 in A549/CDDP cells, the mesenchymal phenotype was reversed and A549/CDDP cells were resensitized to CDDP. Knockdown of Cx43 expression by siRNA induced EMT in A549 cells. These results suggest that the downregulation of $\mathrm{Cx} 43$ expression promotes lung cancer progression and may thus be a therapeutic target for lung cancer.

\section{Materials and methods}

Materials. CDDP, anti-Cx43, anti- $\beta$-actin and dimethylsulfoxide (DMSO) were obtained from Sigma-Aldrich (St. Louis, MO, USA). Cell culture reagents and Lipofectamine ${ }^{\mathrm{TM}} 2000$ were obtained from Invitrogen. Antibodies for E-cadherin, 
vimentin, Slug and Snail were obtained from Cell Signaling Technology (Boston, MA, USA). Secondary antibodies for western blotting were obtained from Amersham Biosciences Corp. (Piscataway, NJ, USA). All other reagents were obtained from Sigma-Aldrich unless stated otherwise.

Overexpression of $C x 43$ and inhibition of $C x 43$ expression by siRNA transfection. Cx43 was expressed in A549/CDDP cells with a pcDNA3.1-Cx43 vector (Shanghai Jima Co. Ltd., Shanghai, China). Cells were transfected with siRNA targeting the human $\mathrm{Cx} 43$ gene (CAGUCUGCCUUUCGU UGUA) or a non-specific control siRNA (NC control in the figures). Transfection into A549 cells was carried out using Lipofectamine 2000 according to the manufacturer's instructions.

MTT assay. MTT assay was used to examine cell viability. Briefly, cells were seeded in a 96-well plate at a density of $5 \times 10^{3} /$ well. After incubation in $5 \% \mathrm{CO}_{2}$ at $37^{\circ} \mathrm{C}$ for $24 \mathrm{~h}$, cells were exposed to different concentrations of CDDP for $48 \mathrm{~h}$. Then $20 \mu 1$ of MTT ( $5 \mathrm{mg} / \mathrm{ml}$ in PBS) was added to each well and cells were incubated for $4 \mathrm{~h}$ at $37^{\circ} \mathrm{C}$. After the medium was removed, the dark blue formazan that formed was dissolved in $200 \mu \mathrm{l}$ DMSO. The absorbance at $570 \mathrm{~nm}$ was measured by a microplate reader (Bio-Rad Laboratories, Hercules, CA, USA). Data presented represent at least three separate repeated experiments.

Western blotting. Western blotting protocols were performed according to our previous study (29). Anti-Cx43, antiE-cadherin, anti-vimentin, anti-Slug and anti-Snail were used. All western blotting exposures were in the linear range of detection, and the intensities of the resulting bands were quantified by Quantity One software on a GS-800 densitometer (Bio-Rad Laboratories).

Wound-healing assay. The cells were seeded into a 6-well plate and incubated until they reached $80 \%$ confluency. A $200-\mu 1$ pipette tip was used to create a wound, and cells were washed twice with serum-free culture media to remove floating cells and then replaced with fresh medium without serum. Cells were subjected to the indicated treatment for $24 \mathrm{~h}$, and cells migrating from the leading edge were photographed at 0 and $24 \mathrm{~h}$.

Matrigel invasion assay. The assay for cell invasion was performed in a 24 -well Transwell unit $(8-\mu \mathrm{m}$ pore size) which was coated with $1 \mathrm{mg} / \mathrm{ml}$ Matrigel matrix as described (30). Briefly, the cells were placed on the Matrigel-coated Transwell (the upper compartment of the invasion chamber) in the presence or absence of drugs. Conditioned medium $(500 \mu \mathrm{l})$ was added to the lower compartment of the invasion chamber. After incubation at $37^{\circ} \mathrm{C}$ for $48 \mathrm{~h}$, cells that had invaded the lower surface of the membrane were fixed with methanol and stained with hematoxylin and eosin. Cells in random fields were counted by light microscopy.

Statistical analysis. Statistical analysis between groups was performed using an unpaired Student's t-test with SigmaPlot 10.0 software (Jandel Scientific, San Rafael, CA, USA). Data

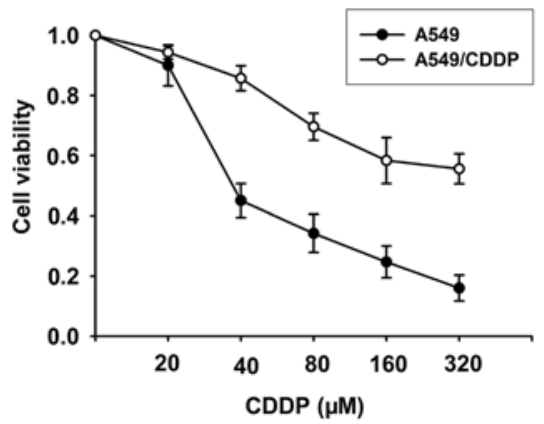

Figure 1. A CDDP-resistant A549 cell line was established. Cells were treated with increasing concentrations of CDDP $(20-320 \mu \mathrm{M})$ for $48 \mathrm{~h}$, and the cell viability was assessed by MTT assay. Results represent the means \pm SEM of 5 independent experiments; bars, SEM. ${ }^{*} \mathrm{P}<0.05$, significantly different from the A549 cells.

are presented as mean \pm SEM. Differences with $\mathrm{P}<0.05$ are considered to have statistical significance.

\section{Results}

Establishing a CDDP-resistant A549 cell line. We generated a CDDP-resistant cell line A549/CDDP by exposing these cells to cisplatin continuously. Cells were exposed to CDDP for more than a year, at an initial concentration of $2.5 \mu \mathrm{M}$ CDDP reaching a final concentration of $40 \mu \mathrm{M}$ CDDP. This cell line was maintained for 6 months. As shown in Fig. 1, the A549/CDDP cell line demonstrated a 5.8-fold higher resistance to CDDP than the A549 cell line.

Acquired resistance of A549/CDDP cells to CDDP induces the cells to undergo EMT. We first observed the morphological changes in A549/CDDP cells. As shown in Fig. 2A, the A549 cells, which were sensitive to CDDP, displayed classical epithelial morphology. In contrast, the A549/CDDP cells, which were resistant to CDDP, showed a spindle-like fibroblastic phenotype and increased formation of pseudopodia (Fig. 2A). These results suggest that the A549/CDDP cells gained a mesenchymal phenotype. To further determine the induction of EMT in A549/CDDP cells, we investigated the expression of EMT markers: epithelial markers such as E-cadherin, and mesenchymal markers such as vimentin, Snail and Slug by western blotting. As shown in Fig. 2B, the expression of E-cadherin was significantly less in the A549/CDDP cells than that in the parental A549 cells. In contrast, the expression levels of vimentin, Snail and Slug were largely increased. These results demonstrated that the acquired resistance to CDDP induced A549/CDDP cells to undergo EMT.

A549/CDDP cells display increased potential for migration and invasion. Cells that have undergone EMT display increased migratory and invasive behaviors. Thus, in the following experiment, we measured the invasive and migratory activity of the cells by Transwell and wound-healing assays. We found that the A549/CDDP cells displayed increased potential for migration and invasion. A549/CDDP cells demonstrated a 3.6-fold increase in invasive capability in the Matrigel-coated membrane when compared with the A549 cells (Fig. 3A). Also, as shown in Fig. 3B, the number of cells migrating across 
A

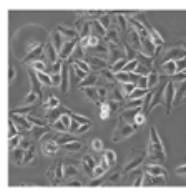

A549

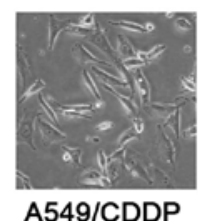

A549/CDDP

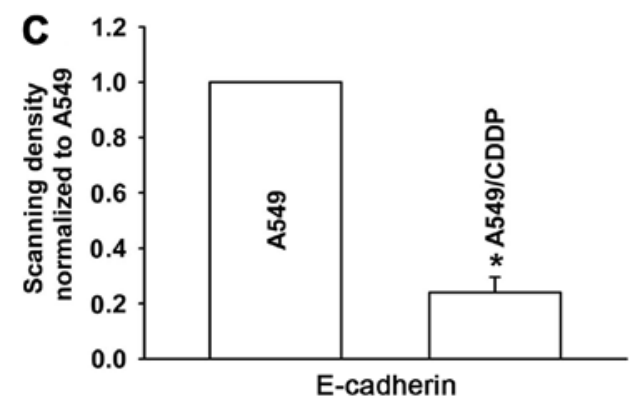

E

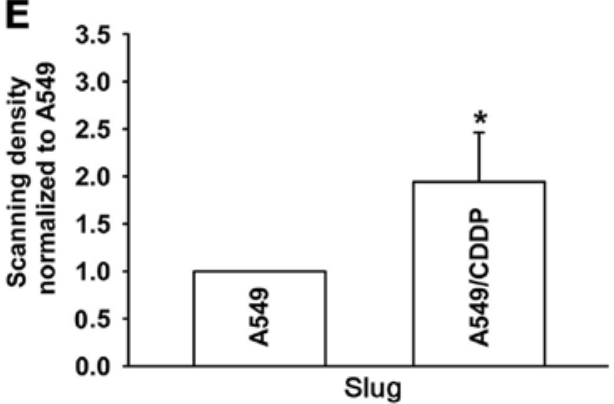

B
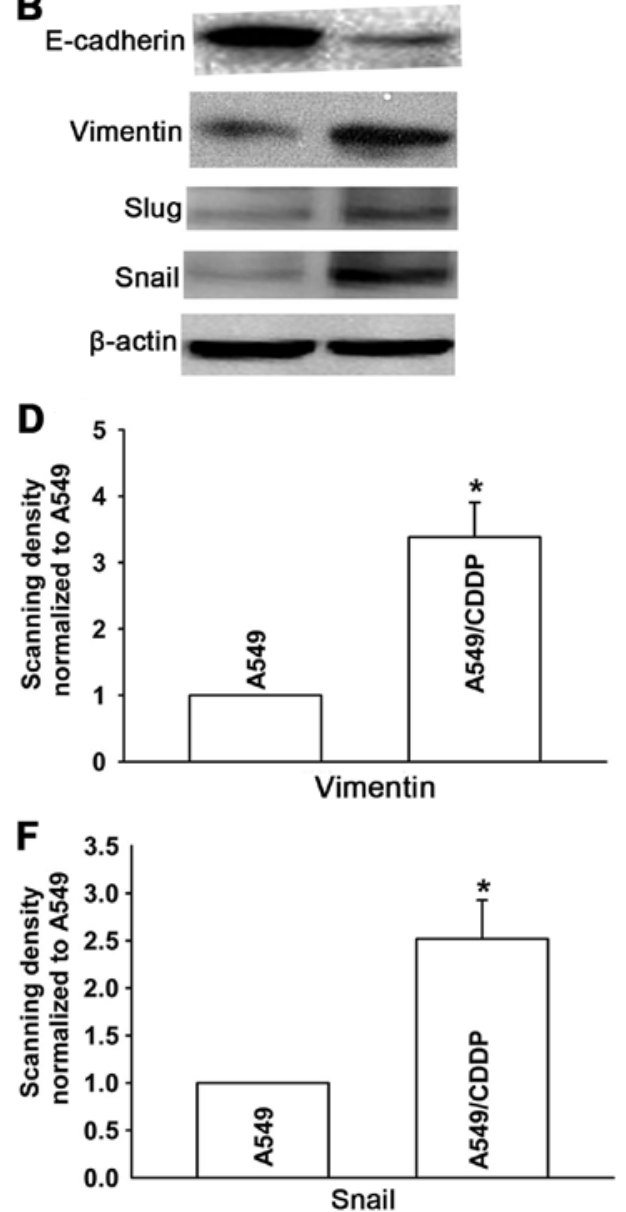

Figure 2. The A549/CDDP cells acquire an EMT phenotype. (A) Images of the morphological changes of A549 and A549/CDDP cells. (B) Western blotting was used to detect the expression of E-cadherin, vimentin, Slug and Snail in the A549 and A549/CDDP cells. (C-F) Bar graphs derived from the densitometric scanning of the blots. Columns, mean from three experiments; bars, SEM. " $\mathrm{P}<0.05$, significantly different from the A549 cells.

A
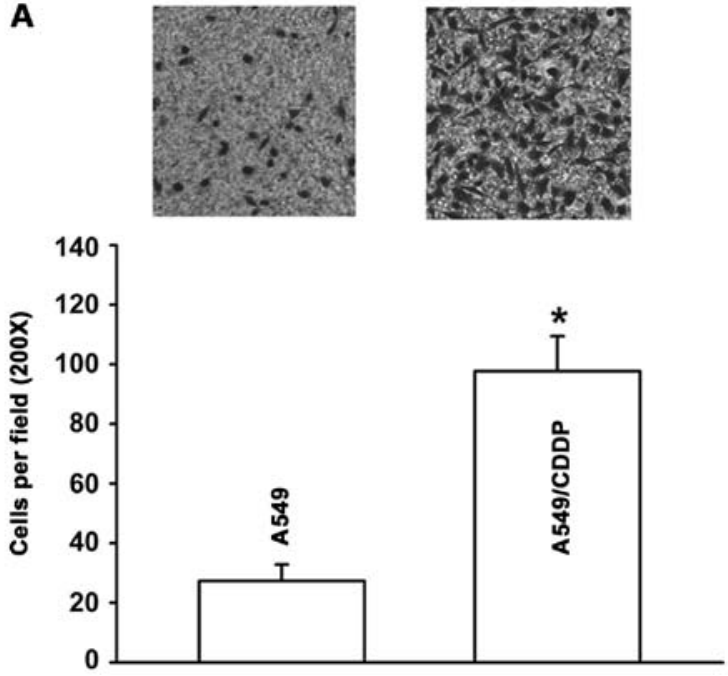

B
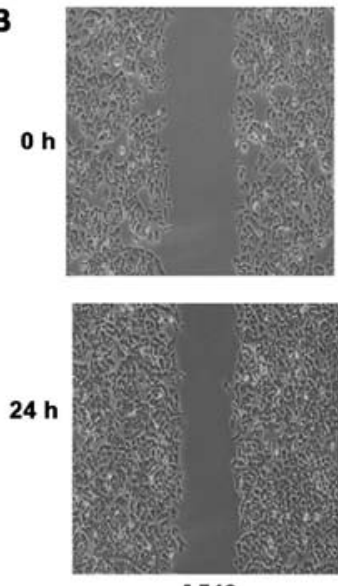
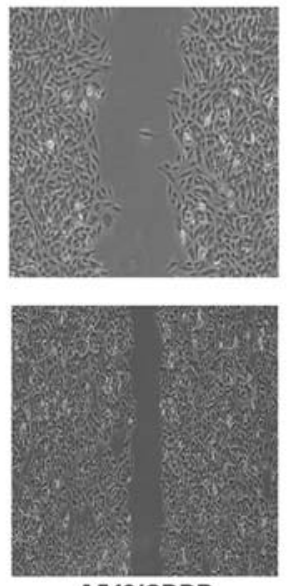

A549/CDDP

Figure 3. A549/CDDP cells display increased potential for migration and invasion. (A) The invasive ability of A549 and A549/CDDP cells was assessed by Matrigel invasion assay. Results represent the means \pm SEM of 3 independent experiments; $\mathrm{P}<0.05$, significantly different from the A549 cells. (B) The migratory ability of A549 and A549/CDDP cells was observed by wound-healing assay wherein cells were scratched with a pipette tip.

the wound in the A549/CDDP cells was significantly increased relative to the A549 cells, suggesting that A549/CDDP cells showed enhanced migratory activity.
A549/CDDP cells show decreased expression of Cx43. To explore the effect of $\mathrm{Cx} 43$ on the resistance of lung adenocarcinoma cells to CDDP, we investigated the $\mathrm{Cx} 43$ expression 


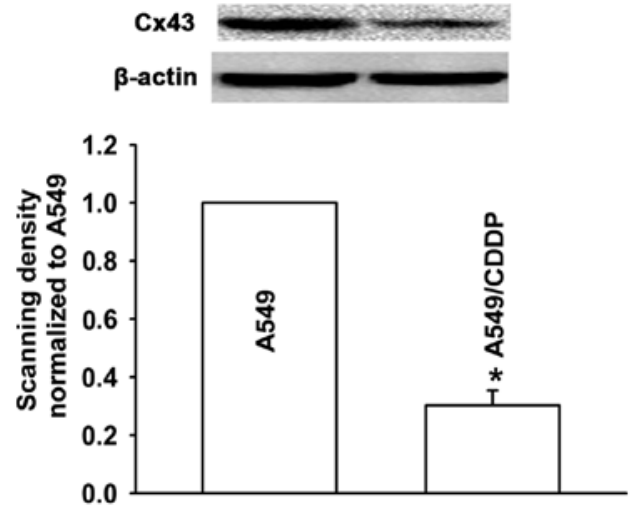

Figure 4. A549/CDDP cells show decreased expression of Cx43. Western blotting was used to detect the expression of Cx43 in A549 and A549/CDDP cells. Bar graphs derived from the densitometric scanning of the blots. Columns, mean from three experiments; bars, SEM. " $\mathrm{P}<0.05$, significantly different from A549 cells.

A
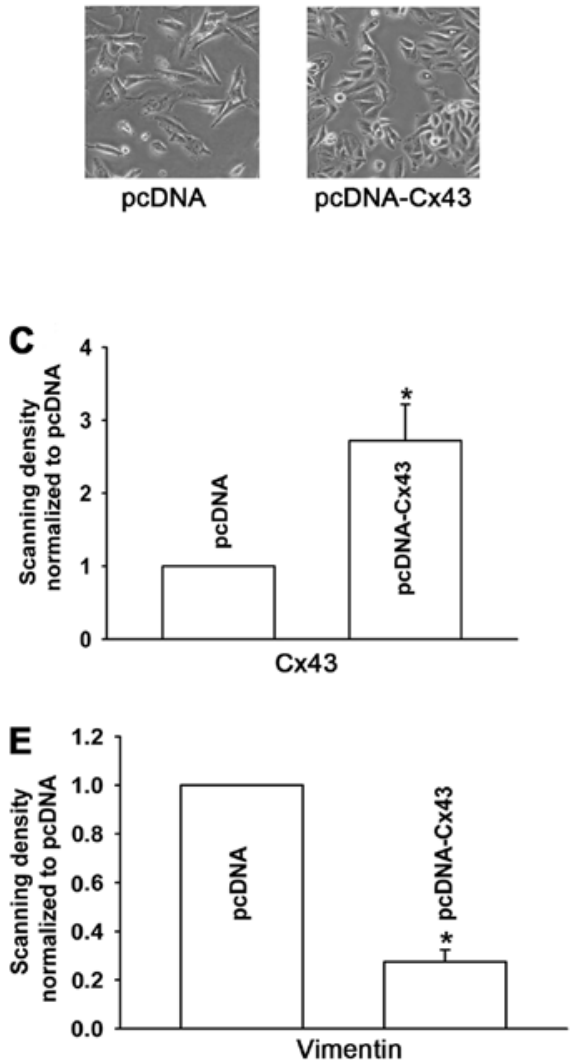

B
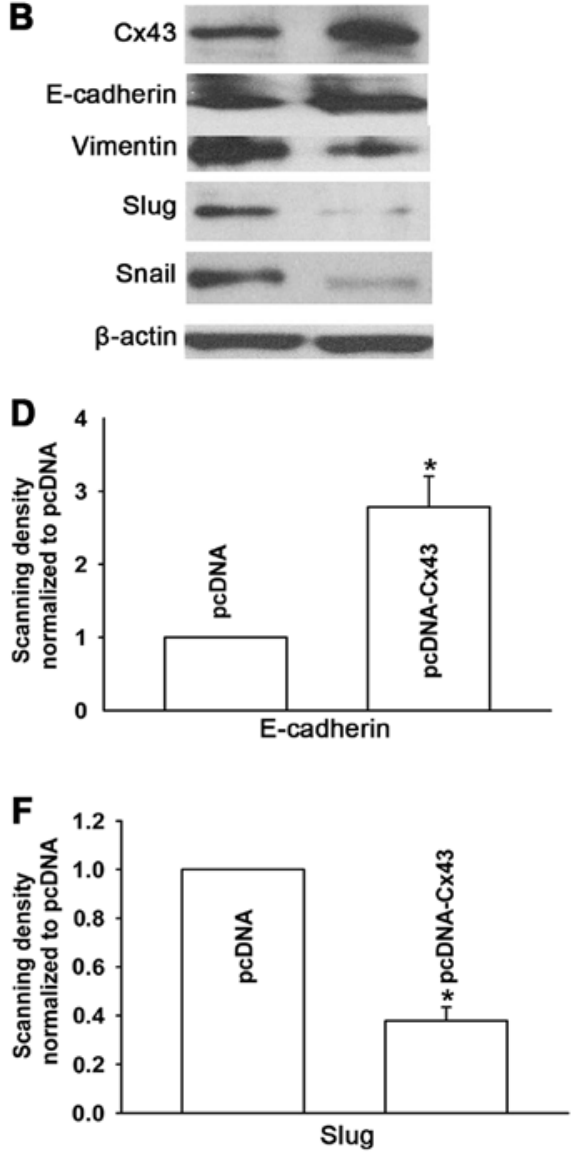

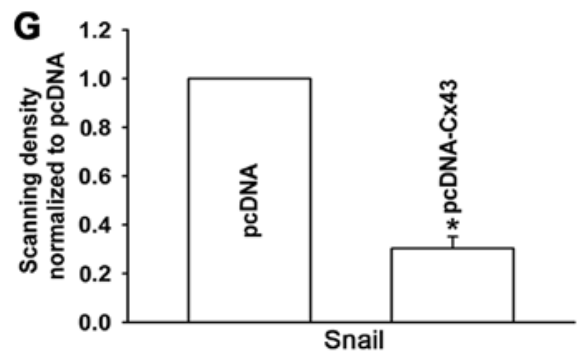

Figure 5. Overexpression of Cx43 reverses EMT in A549/CDDP cells. (A) Images of morphological changes of A549/CDDP cells following transfection by pcDNA-Cx43. (B) The effect of Cx43 overexpression on the expression of E-cadherin, vimentin, Slug and Snail was determined by western blotting in A549/CDDP cells. (C-G) Bar graphs derived from the densitometric scanning of the blots. Columns, mean from three experiments; bars, SEM. "P<0.05, significantly different from pcDNA-transfected group. 

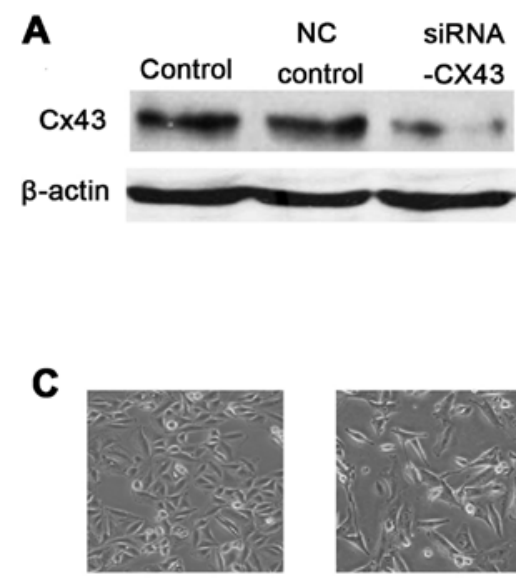

NC control

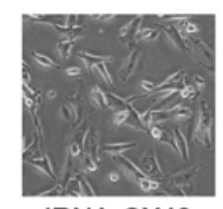

SiRNA-CX43
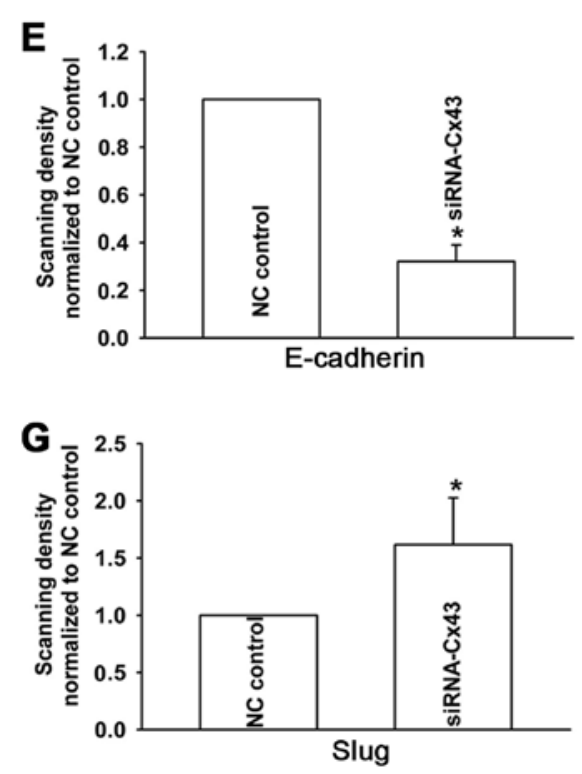

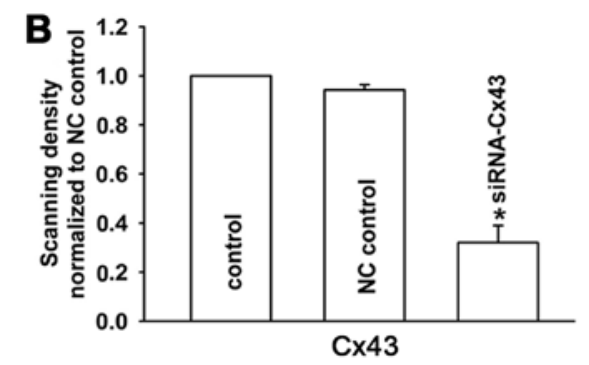

D
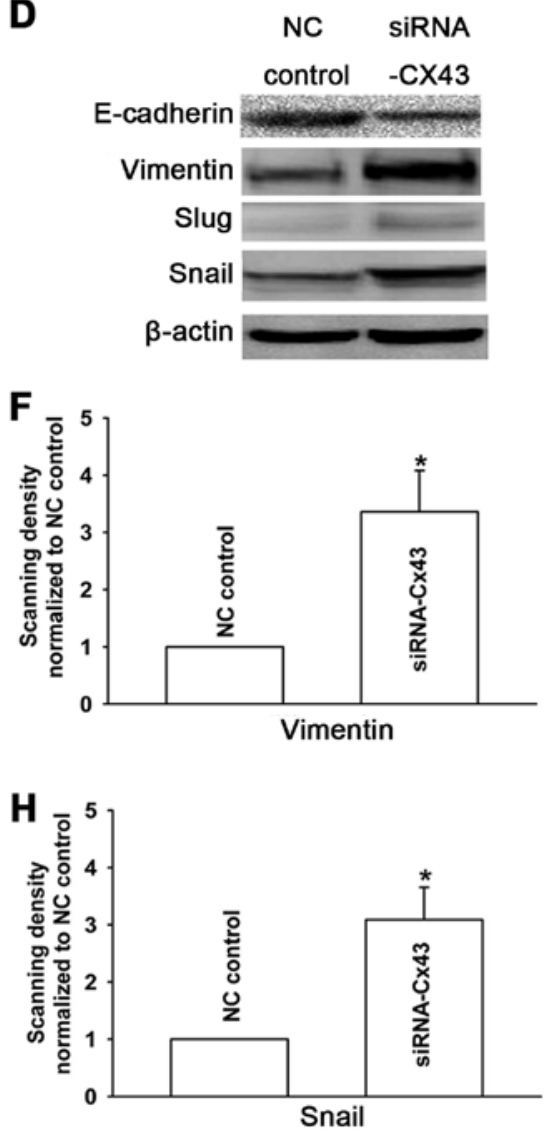

Figure 6. Knockdown of Cx43 expression induces EMT in A549 cells. (A) Expression of Cx43 in A549 cells following treatment with siRNA-Cx43. (B) Bar graph derived from the densitometric scanning of the blots. Columns, mean from three experiments; bars, SEM. "P<0.05, significantly different from NC control. (C) Images of morphological changes in the A549 cells following treatment with siRNA-Cx43. (D) The effect of Cx43 knockdown on the expression of E-cadherin, vimentin, Slug and Snail was determined by western blotting in A549 cells. (E-H) Bar graphs derived from the densitometric scanning of the blots. Columns, mean from three experiments; bars, SEM. ${ }^{*} \mathrm{P}<0.05$, significantly different from NC control.

level in the A549 cells and A549/CDDP cells by western blotting. The expression level of Cx43 in the A549/CDDP cells was significantly decreased when compared with that in the A549 cells (Fig. 4). This suggests that Cx43 may be involved in CDDP-induced EMT in human lung cancer.

CX43 is involved in the regulation of CDDP-induced EMT Overexpression of Cx43 reverses EMT in A549/CDDP cells. Based on the results described above, we hypothesized that Cx43 regulates EMT in A549 cells. Thus, the downregulation of Cx43 may initiate EMT during the development of the resistance of A549 cells to CDDP. To directly investigate the role of Cx43 in CDDP-induced EMT, we manipulated Cx43 expression levels in two ways: overexpression of $\mathrm{Cx} 43$ by transfection of pcDNA-Cx43 and knockdown of Cx43 expression with siRNA-Cx43. Firstly, we overexpressed Cx43 by transfection of pcDNA-Cx43 in A549/CDDP cells. Western blotting was used to confirm this effect (Fig. 5B). Consistent with the previous experiment (Fig. 2), the morphological changes and the marker proteins associated with EMT (epithelial marker such as E-cadherin, and mesenchymal markers such as vimentin, Snail and Slug) were observed in the subsequent experimental study. Fig. 5A shows that the pcDNA-transfected cells exhibited an elongated fibroblast-like morphology, whereas Cx43-A549/CDDP cells, which had a high Cx43 expression level, displayed epithelial morphology. Moreover, compared with the pcDNA-transfected cells, the expression level of epithelial marker E-cadherin was significantly increased, while levels of mesenchymal markers vimentin, Snail and Slug were decreased upon overexpression of $\mathrm{Cx} 43$ in the A549/CDDP cell line (Fig. 5B). These results demonstrate that upregulation of $\mathrm{Cx} 43$ by pcDNA-Cx43 converted EMT to mesenchymal-epithelial transition (MET) in the A549/CDDP cells. 
A

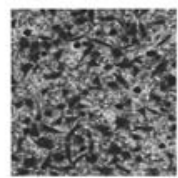

pcDNA

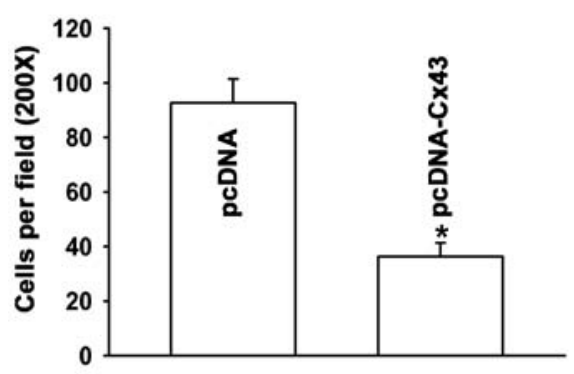

B

$\mathbf{~ h ~}$

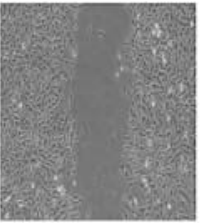

$24 \mathrm{~h}$

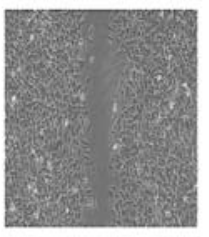

pcDNA

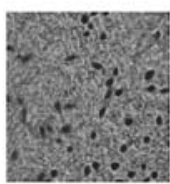

pcDNA-CX43
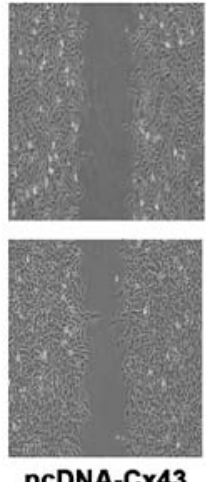

C

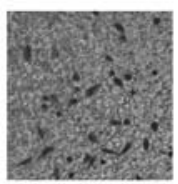

NC control

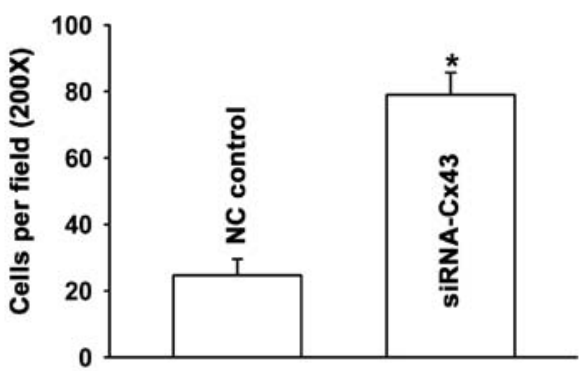

D

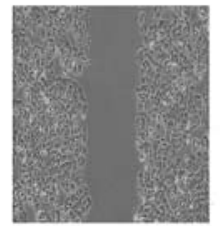

$24 \mathrm{~h}$

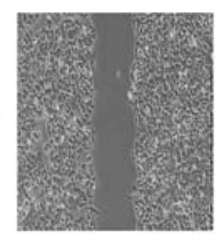

NC control
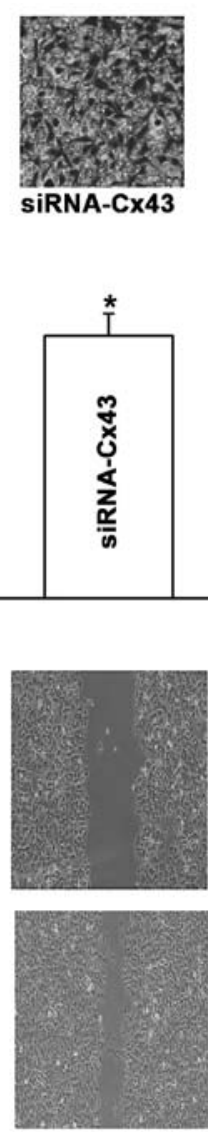

SiRNA-Cx43

Figure 7. Cx43 regulates the capability of invasion and migration in human adenocarcinoma cells. (A) The invasive ability of A549/CDDP cells following transfection by pcDNA-Cx43. Results represent the means \pm SEM of 3 independent experiments; ${ }^{*} \mathrm{P}<0.05$, significantly different from the pcDNA-transfected group. (B) The effect of Cx43 overexpression on the migratory ability of A549/CDDP cells by wound-healing assay. (C) The invasive ability of A549 cells following treatment with siRNA-Cx43. Results represent the means \pm SEM of 3 independent experiments; ${ }^{*} \mathrm{P}<0.05$, significantly different from the NC control. (D) The effect of Cx43 knockdown on the migratory ability of A549 cells by wound-healing assay.
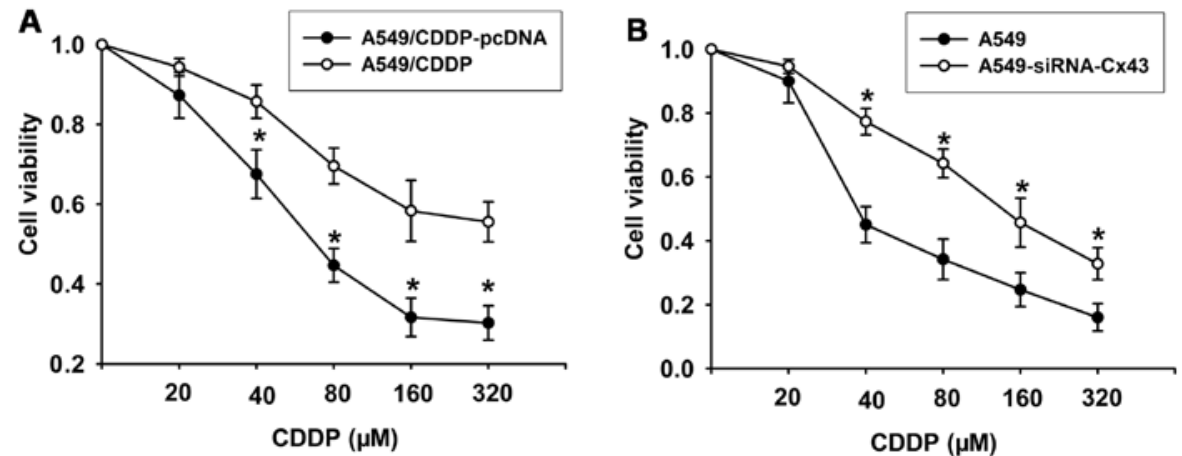

Figure 8. Cx43 regulates the cytotoxicity of CDDP in human adenocarcinoma cells. (A) Overexpression of Cx43 reduced cellular resistance to CDDP in the A549/CDDP cells. The effect of Cx43 overexpression on the cytotoxicity of CDDP (20-320 $\mu \mathrm{M})$ in the A549/CDDP cells was detected by MTT assay. Results represent the means \pm SEM of 5 independent experiments; bars, SEM. "P $<0.05$, significantly different from the A549/CDDP cells. (B) Cx43 knockdown reduced CDDP sensitivity in the A549 cells. The effect of Cx43 knockdown on the cytotoxicity of CDDP $(20-320 \mu \mathrm{M})$ in the A549 cells was detected by MTT. Results represent the means \pm SEM of 5 independent experiments; bars, SEM. "P $<0.05$, significantly different from the A549 cells.

Knockdown of Cx43 expression induces EMT in A549 cells. To further verify whether the EMT-associated phenotypes were specifically regulated by $\mathrm{Cx} 43$, we downregulated Cx43 expression in A549 cells which were sensitive to CDDP using siRNA (Fig. 6A and B). The results showed that A549 cells underwent EMT. Compared with the NC control cells, the cells displayed a spindle-like fibroblastic phenotype (Fig. 6C), and the expression of E-cadherin was markedly decreased, while the expression levels of vimentin, Snail and Slug were upregulated in A549 cells transfected with Cx43 siRNA (Fig. 6D). These results suggest that $\mathrm{Cx} 43$ is involved in the regulation of CDDP-induced EMT in human lung cancer cells. 
Cx43 regulates invasive and migratory properties of cells. To further investigate the effect of Cx43 on EMT in human lung cancer, we investigated the invasive and migratory properties of the cells. The results in Fig. 7A and B revealed that when A549/CDDP cells were transfected with Cx43, the capability of these cells to migrate and invade were obviously reduced relative to the pcDNA-transfected cells. In contrast, A549 cells transfected with CX43 siRNA showed significant enhancement in their invasive and migratory properties (Fig. 7C and D). These results provide further evidence that $\mathrm{Cx} 43$ is involved in the regulation of CDDP-induced EMT in human lung cancer cells.

Cx43 regulates CDDP-induced cytoxicity in human adenocarcinoma cells. To observe the effect of $\mathrm{Cx} 43$ on the cytotoxic effect of CDDP, we manipulated Cx43 expression in two ways: overexpression of $\mathrm{Cx} 43$ by transfection of pcDNA-Cx43 in A549/CDDP cells and knockdown of Cx43 expression with siRNA-Cx43 in A549 cells. The results showed that compared with the pcDNA-transfected cells, overexpression of $\mathrm{Cx} 43$ in the A549/CDDP cell line significantly reversed resistance of the cells to CDDP (Fig. 8A). Conversely, knockdown of $\mathrm{Cx} 43$ expression with siRNA-Cx43 resulted in insensitivity of A549 cells to CDDP (Fig. 8B). These results suggest that downregulation of $\mathrm{Cx} 43$ which induces EMT may underlie the resistance of A549 cells to CDDP.

\section{Discussion}

Emerging evidence suggests that chemo-resistance is associated with the acquisition of EMT in cancer cells $(3-5,31,32)$. For example, gemcitabine-resistant pancreatic cancer (31), tamoxifen-resistant MCF7 breast cancer (5), oxaliplatin-resistant colorectal cancer (4), paclitaxel-resistant ovarian cancer (3) and gefitinib-resistant lung cancer cells (32) acquire EMT characteristics. Novel research has demonstrated that targeting EMT is a promising new therapeutic strategy (33-36). Tan et al (33) reported that Par-4 downregulation confers CDDP resistance in pancreatic cancer cells by inducing PI3K/Akt pathwaydependent EMT; knockdown of miR-134/487b/655 inhibited the EMT process and reversed TGF- $\beta 1$-induced resistance to gefitinib in lung adenocarcinoma cells (34); downregulation of the PDGF-D pathway reversed EMT in gemcitabine-resistant hepatocellular cancer cells (35); and knockdown of Snail and Slug reversed CDDP resistance in ovarian cancer (36). Thus, regulating EMT is a new way to overcome drug resistance in cancer cells and to promote a better treatment outcome for cancer patients.

Consistent with the above studies, when the human lung adenocarcinoma cell line A549 became resistant to CDDP, these cells underwent EMT with significant morphological changes such as acquisition of a classical mesenchymal phenotype, downregulation of epithelial marker E-cadherin, and upregulation of mesenchymal markers vimentin, Snail and Slug. However, the exact mechanism underlying the CDDPinduced EMT phenotype of A549 cancer cells is still unclear.

Cx43 has been reported to play important roles in cancer development, cell proliferation, apoptosis, invasion and metastasis in lung cancer (20-26). A study showed that concurrent reduction in the expression of $\mathrm{Cx} 43$ and E-cadherin may contribute to the development of lung cancer. For example, Cx43 may induce E-cadherin expression and inhibit cell proliferation and progression of lung cancer (20). Another report also confirmed that transfection of $\mathrm{Cx} 43$ induced E-cadherin overexpression (23). Since E-cadherin is a typical epithelial marker, based on these reports, $\mathrm{Cx} 43$ may influence lung cancer development by regulating EMT. Indeed, the expression level of Cx43 was significantly less in A549/CDDP cells than that in the parental A549 cells. To further investigate the effect of Cx43 expression on CDDP-induced EMT in the human adenocarcinoma cell line, firstly, Cx43 was overexpressed in A549/CDDP cells. This resulted in A549/CDDP cells displaying classical epithelial morphology, increased E-cadherin expression, decreased expression of mesenchymal markers, reduced invasive and migratory activity and resensitization to CDDP. This suggests that overexpression of Cx43 reversed CDDP-induced EMT and enhanced the cytotoxicity of CDDP. Secondly, when Cx43 expression was knocked down in A549 cells sensitive to CDDP exposure, the cells underwent EMT. Thus, the present study is the first report to show that Cx43 is involved in the regulation of CDDP-induced EMT in human lung adenocarcinoma cells.

Cx43 could sensitize cancer cells to chemotherapeutic agents, including CDDP $(37,38)$. Although the mechanisms underlying these effects are still not clear, we can speculate the following scenarios: i) $\mathrm{Cx} 43$ could improve cell resistance to CDDP which may be mediated by the suppression of Src activity (37); ii) Cx43 could enhance the cytotoxic effect of various chemotherapeutic agents (etoposide, pacitaxel, doxorubicin) by promoting apoptosis (39); and iii) $\mathrm{Cx} 43$ enhanced the efficiency of CDDP in tumor testicular cells by gap junctional intercellular communication (GJIC)-mediated toxic bystander effects (38). The present study provides the first evidence that Cx43 enhances the cytotoxicity of CDDP by regulating EMT in human lung cancer cells.

In summary, the present study showed that $\mathrm{Cx} 43$ plays a critical role in promoting lung cancer progression and CDDP resistance by regulating EMT. Overexpression of Cx43 in CDDP-resistant A549 cells reversed EMT to MET and enhanced the cytotoxity of CDDP. Thus, Cx43 may be a potential target for overcoming CDDP resistance in lung cancer therapy.

\section{Acknowledgements}

The present study was supported by the China Postdoctoral Science Foundation (no. 20090461139), the National Natural Science Foundation of China (no. 81001457) and the Foundation of Bengbu Medical College (no. Bykf13A11 and no. Byycx1329).

\section{References}

1. Siegel R, Naishadham D and Jemal A: Cancer statistics, 2012. CA Cancer J Clin 62: 10-29, 2012.

2. Ota S, Ishii G, Goto K, et al: Immunohistochemical expression of BCRP and ERCC1 in biopsy specimen predicts survival in advanced non-small-cell lung cancer treated with cisplatin-based chemotherapy. Lung Cancer 64: 98-104, 2009.

3. Kajiyama H, Shibata K, Terauchi M, et al: Chemoresistance to paclitaxel induces epithelial mesenchymal transition and enhances metastatic potential for epithelial ovarian carcinoma cells. Int J Oncol 31: 277-283, 2007. 
4. Yang AD, Fan F, Camp ER, et al: Chronic oxaliplatin resistance induces epithelial-to-mesenchymal transition in colorectal cancer cell lines. Clin Cancer Res 12: 4147-4153, 2006.

5. Hiscox S, Jiang WG, Obermeier K, et al: Tamoxifen resistance in MCF7 cells promotes EMT-like behaviour and involves modulation of $\beta$-catenin phosphorylation. Int J Cancer 118 : 290-301, 2006

6. Hiscox S, Morgan L, Barrow D, Dutkowskil C, Wakeling A and Nicholson RI: Tamoxifen resistance in breast cancer cells is accompanied by an enhanced motile and invasive phenotype: inhibition by gefitinib ('Iressa', ZD1839). Clin Exp Metastasis 21: 201-212, 2004

7. Chang CJ, Chao $\mathrm{CH}$, Xia W, et al: p53 regulates epithelialmesenchymal transition and stem cell properties through modulating miRNAs. Nat Cell Biol 13: 317-323, 2011.

8. Thiery JP, Acloque H, Huang RY and Nieto MA: Epithelialmesenchymal transitions in development and disease. Cell 139: 871-890, 2009.

9. Voulgari A and Pintzas A: Epithelial-mesenchymal transition in cancer metastasis: mechanism, markers and strategies to overcome drug resistance in the clinic. Biochim Biophys Acta 1796: 75-90, 2009

10. Hugo H, Ackland ML, Blick T, et al: Epithelial-mesenchymal and mesenchymal-epithelial transitions in carcinoma progression. J Cell Physiol 213: 374-383, 2007.

11. Min C, Eddy SF, Sherr DH and Sonenshein GE: NF- $\kappa$ B and epithelial to mesenchymal transition of cancer. J Cell Biochem 104: 733-744, 2008

12. Proksch E, Brandner JM and Jensen JM: The skin: an indispensable barrier. Exp Dermatol 17: 1063-1072, 2008.

13. Laird DW: Life cycle of connexins in health and disease. $\mathrm{J}$ Biochem 394: 527-543, 2006.

14. Cronier L, Crespin S, Strale PO, Defamie N and Mesnil M: Gap junctions and cancer: new functions for an old story. Antioxid Redox Signal 11: 323-338, 2009.

15. Plante I, Stewart MK, Barr K, Allan AL and Laird DW: Cx43 suppresses mammary tumor metastasis to the lung in a $\mathrm{Cx} 43$ mutant mouse model of human disease. Oncogene 30: 1681-1692, 2011.

16. Ogawa K, Pitchakarn P, Suzuki S, et al: Silencing of connexin 43 suppresses invasion, migration and lung metastasis of rat hepatocellular carcinoma cells. Cancer Sci 103: 860-867, 2012.

17. Willecke K, Eiberger J, Degen J, et al: Structural and functional diversity of connexin genes in the mouse and human genome. Biol Chem 383: 725-737, 2002

18. Okuma A, Kuraoka A, Iida H, Inai T, Wasano K and Shibata Y: Colocalization of connexin 43 and connexin 45 but absence of connexin 40 in granulosa cell gap junctions of rat ovary. J Reprod Fertil 107: 255-264, 1996

19. Risley MS, Tan IP, Roy C and Saez JC: Cell-, age- and stage dependent distribution of connexin43 gap junctions in testes. $\mathrm{J}$ Cell Sci 103: 81-96, 1992.

20. de Oliveira KD, Tedardi MV, Cogliati B and Dagli ML: Higher incidence of lung adenocarcinomaa induced by DMBA in Cx43 heterozygous knockout mice. Biomed Res Int 2013: 618475, 2013. doi: $10.1155 / 2013 / 618475$

21. Zhao W, Han HB and Zhang ZQ: Suppression of lung cancer cell invasion and metastasis by $\mathrm{Cx} 43$ involves the secretion of follistatin-like 1 mediated via histone acetylation. Int J Biochem Cell Biol 43: 1459-1468, 2011.

22. Xu HT, Li QC, Zhang YX, et al: Cx43 recruits E-cadherin expression and inhibits the malignant behaviour of lung cancer cells. Folia Histochem Cytobiol 46: 315-321, 2008.

23. Zhang YX, Xu HT, Qi FJ and Wang EH: Expression of Cx43 in lung cancer and its correlation with E-cadherin. Zhonghua Bing Li Xue Za Zhi 35: 339-343, 2006 (In Chinese).
24. Chen Q, Qian B, Yang L and Jiang Z: The expression of Cx43 protein in non-small cell lung cancer tissues and its clinical significance. Zhongguo Fei Ai Za Zhi 6: 272-274, 2003 (In Chinese).

25. Cesen-Cummings K, Fernstrom MJ, Malkinson AM and Ruch RJ: Frequent reduction of GJIC and Cx43 expression in human and mouse lung carcinoma cells. Carcinogenesis 19: 61-67, 1998

26. Lin Z, Zhang $\mathrm{Z}$ and Wang $\mathrm{N}$ : Inhibition of in vivo growth of lung carcinoma cells after transfetion with gap junction gene $C x 43$. Zhonghua Zhong Liu Za Zhi 19: 253-255, 1997 (In Chinese).

27. Du G, Yang Y, Zhang Y, et al: Thrombocytosis and immunohistochemical expression of connexin43 at diagnosis predict survival in advanced non-small-cell lung cancer treated with cisplatin-based chemotherapy. Cancer Chemother Pharmacol 71: 893-904, 2013.

28. Zhang L, Sharma S, Hershman JM, Brent GA, Dubinett SM and Huang M: Iodide sensitizes genetically modified non-small cell lung cancer cells to ionizing radiation. Cancer Gene Ther 13: 74-81, 2006.

29. Yu ML, Zhang CL, Yuan DD, Tong XH and Tao L: Panax notoginseng saponins enhances the cytotoxicity of cisplatin via increasing gap junction intercellular communication. Biol Pharm Bull 35: 1230-1237, 2012.

30. Kim KS, Yao L, Lee YC, et al: Hyul-Tong-Ryung suppresses PMA-induced MMP-9 expression by inhibiting AP-1-mediated gene expression via ERK1/2 signaling pathway in MCF-7 human breast cancer cells. Immunopharmacol Immunotoxicol 32: 600-606, 2010

31. Wang Z, Li Y, Kong D, et al: Acquisition of epithelial-mesenchymal transition phenotype of gemcitabine-resistant pancreatic cancer cells is linked with activation of the notch signaling pathway. Cancer Res 69: 2400-2407, 2009.

32. Rho JK, Choi YJ, Lee JK, et al: Epithelial to mesenchymal transition derived from repeated exposure to gefitinib determines the sensitivity to EGFR inhibitors in A549, a non-small cell lung cancer cell line. Lung Cancer 63: 219-226, 2009.

33. Tan J, You Y, Xu T, et al: Par-4 downregulation confers cisplatin resistance in pancreatic cancercells via PI3K/Akt pathwaydependent EMT. Toxicol Lett 224: 7-15, 2013.

34. Kitamura K, Seike M, Okano T, et al: MiR-134/487b/655 cluster regulates TGF- $\beta$-induced epithelial-mesenchymal transition and drug resistance to gefitinib by targeting MAGI2 in lung adenocarcinoma cells. Mol Cancer Ther 13: 444-453, 2014.

35. Wu Q, Wang R, Yang Q, et al: Chemoresistance to gemcitabine in hepatoma cells induces epithelial-mesenchymal transition and involves activation of PDGF-D pathway. Oncotarget 4: 1999-2009, 2013

36. Haslehurst AM, Koti M, Dharsee M, et al: EMT transcription factors snail and slug directly contribute to cisplatin resistance in ovarian cancer. BMC Cancer 12: 91, 2012. doi: 10.1186/14712407-12-91, 2012.

37. Sato H, Iwata H, Takano Y, et al: Enhanced effect of $\mathrm{Cx} 43$ on cisplatin-induced cytotoxicity in mesothelima cells. J Pharmacol Sci 110: 466-475, 2009.

38. Hong X, Wang Q, Yang Y, et al: Gap junctions propagate opposite effects in normal and tumor testicular cells in response to cisplatin. Cancer Lett 317: 165-171, 2012.

39. Huang RP, Hossain MZ, Huang R, Gano J, Fan Y and Boynton AL: Connexin 43 (cx43) enhances chemotherapy-induce apoptosis in human glioblastoma cells. Int J Cancer 92: 130-138, 2001. 